\title{
Green Computing in Software Defined Social Internet of Vehicles
}

\author{
Neetesh Kumar Member, IEEE, Rashmi Chaudhry, Omprakash Kaiwartya Senior Member, IEEE, Neeraj Kumar \\ Senior Member, IEEE, Syed Hassan Ahmed Senior Member, IEEE
}

\begin{abstract}
Social Internet of Vehicles (SIoV) is an evolving vehicular networking framework integrating the next generation smart devices with vehicular communications. Green computing and communication under disruptive vehicular environment is one of the challenging tasks for enabling SIoV. In this context, green traffic data dissemination in SIoV environments is modelled as an NP-hard problem focusing on heterogeneous traffic data, transmission distance from next generation smart devices and probabilistic delay in transmissions due to disruptive vehicular environment. An adopted meta-heuristic solution namely TwoWay Particle Swarm Optimization (TWPSO) is developed for the green traffic data dissemination problem in SIoV considering software defined vehicular network architecture. Extensive simulation experiments were performed to assess the performance of TWPSO as compared to the state-of-the-art techniques. The critical analysis of the comparative results attest the green computing oriented benefits of TWPSO under real SIoV environments.
\end{abstract}

Index Terms-Green computing, Social Internet of vehicles, Software defined vehicular networking.

\section{INTRODUCTION}

$\mathbf{I}$ NTELLIGENT Transportation System (ITS) has been enriching towards sensors enabled smart devices attached with roads, traffic lights, sign boards, and vehicles with computing and communication capabilities [1]. The sensor enabled smart traffic environments is transforming the traditional vehicular networking towards Social Internet of connected Vehicles (SIoV) [2]. Recent advancements in SIoV have given rise to the smart services including on-road traffic information, nearby restaurants, gas stations, hospitals and lots of other utility services [3]. Technically, SIoV is a dynamic mobile network that empowers information dissemination among vehicles, battery powered smart devices and cloud or Fog enabled servers [4] through advancements in communication's generations. SIoV is an embryonic vehicular cyber-physical system which extends vehicular adhoc networks (VANETs), Internet of Things (IoT) and fog or edge servers in combination [5].

SIoV addresses on-road traffic safety and efficiency issues in an complex and adaptive vehicular networking environment

This work was supported by Science and Engineering Research Board (SERB), Government of India under Grant no. EEQ/2019/000182. (Corresponding Author: Neetesh Kumar)

N. Kumar is with Indian Institute of Technology Roorkeev(IITR), Uttrakhand 247667, India (e-mail: neeteshiitr@gmail.com).

R. Chaudhry is with International Institute of Information Technology, Naya Raipur, India-493661

O. Kaiwartya is with Nottingham Trent University, Nottingham, UK (email: omprakash.kaiwartya@ntu.ac.uk).

N. Kumar is with King Abdul Aziz University Saudi Arabia (email:neeraj.kumar.in@ieee.org).

SH. Ahmed is with JMA Wireless, USA (e-mail:sh.ahmed@ieee.org).
[6]. Commercialization of SIoV includes addressing the issues related to cost of network management, frequent change in its topology [7], unreliable Internet service, incompatibility with personal devices, heterogeneous environment, frequent change in the network architecture and poor management of battery powered smart devices in traffic environment [8].

Software defined SIoV architecture decouples vehicular network into data and control planes [9]. In the proposed architecture, the sensor enabled smart devices in traffic environment are considered in data plane which offers the collection of traffic data from the environment and send it to the control plane nodes. The vehicles are considered into control plane nodes which provides forwarding of traffic data to the cloud or edge enabled control server (CS). Further, it enables network control to become directly programmable and the underlying infrastructure to be abstracted based the requirement of traffic applications and network services. It permits the realization of flexible vehicular edge or cloud framework as a CS entity [10]. The rest of the control nodes including smart devices and RSUs in traffic environments can also be treated as Software defined Vehicular Nodes (SVN), with the flexibility of changing the role of the control nodes via programmable control from the CS [11].

Green computing in software defined SIoV environments is significant for considering the heterogeneous and disruptive vehicular networking environments [12]. Effective energy utilization in SVN is critical for durable distributed operation of various smart devices in traffic environments. The energy utilization in SVN can be defined in terms of communication disruption centric delay, dynamic transmission distance, and heterogeneous traffic data in SIoV environments. In this context, this paper proposes a framework for enabling green computing in software defined social internet of vehicles focusing on next generation smart devices attached traffic environment. The major contributions of the paper can be summarized in following folds:

- Firstly, a system model for software defined social Internet of Vehicles is presented by decoupling vehicular networking architecture into data and control planes.

- Secondly, green computing in social Internet of vehicles is modeled as an NP-hard optimization problem considering the constraints of disruptive vehicular environments.

- Thirdly, an adapted meta-heuristic solution Two-Way Particle Swarm Optimization (TWPSO) is developed focusing on software defined vehicular networking architecture.

- Finally, a case study is performed to comparatively assess 


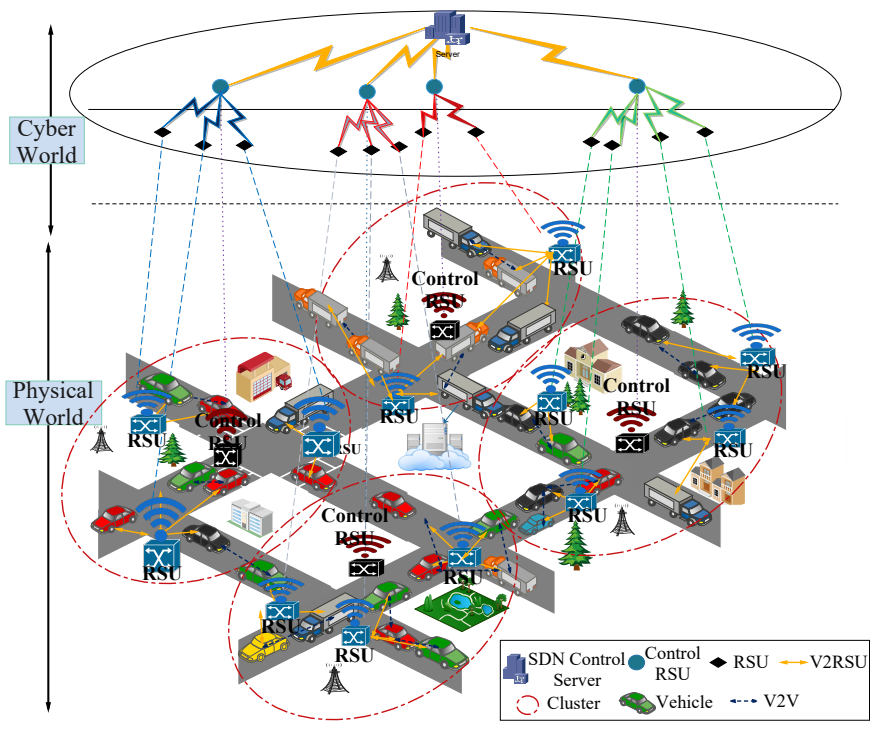

Fig. 1: Cyber-Physical world interactions in SIoV environment illustrating hierarchical network architecture with SDN environment

the green computing centric performance benefits of TWPSO with the state-of-the-art heuristic solutions.

The rest of the paper is organized as follows. The related literature on SIoV is critically explored in Section II. The details of the proposed green computing framework in SIoV environment is presented in Section III. Section IV discusses a case study with in-depth analysis of experimental results, followed by the concluding remarks presented in Section V.

\section{LiterATURE SURVEY}

Intelligent Transportation System has been emerged as the primary focused research area in smart cities [13]. Inclusion of the V2V and V2I communication in ITS has enabled SIoV to deal with traffic management, disaster management, intelligent traffic surveillance and other emergency services [2]. SIoVs need continuous communication among their specified group of vehicles associated with RSUs/OBUs [3], therefore, data routing plays a significant role in SIoVs to act in real-time and dynamic scenarios. Generally, in SIoVs, one entity needs to communicate with multiple entities; therefore, an effective architecture with multicast routing feasibility is demanded. Multicast routing is necessity to establish SIoV network, it has been traditionally used in wireless Ad-hoc sensors networks [14] and Vehicular Ad-hoc Networks [15].

Considering Vehicular network dynamics requirements, SDSIoV in transport management has progressed as a network archetype to enable the vehicular network with dynamic, robust, reliable with auto configurable. Following this, Sadio et al. [16] came up with an architecture which consists of four layers to provide programmability, flexibility, scalability and global knowledge. These layers include cloud, SDN controller, fog and vehicles. Further, authors [15] utilized the SDN controller for routing packets from server to VANET. To predict the trajectory of the vehicles an architecture was also proposed, it performed multicast and scheduling with minimal cost and optimal for delay-constraint applications [4]. SD-SIoV has also been proposed for wireless networks to add tractability to integrate exponentially growing wireless traffic and to acclimate network configurations [17]. Despite of feasibility of SD-SIoV to manage vehicular network with numerous benefits, energy consumption in SD-SIoV routing remains a matter of concern. Few methods for energy aware routing in deterministic scenarios have been discussed in [18]. An energy aware routing protocol in vehicular networks with the help of openflow switches and SDN controller. Further, bandwidth and deadline constraints are considered while forwarding the packets to other nodes, however, authors did not account the green communication requirement [19] [20]. As the battery life of sensor nodes in SD-SIoV is limited, therefore, this work explored a requirement of energy efficient or green multicast routing algorithm in SIoVs to maximize the vehicular network lifetime during the continuous data communication on auto-configured and most demanding SDSIoV enabled architecture.

\section{Software Defined Social Internet of Vehicles}

\section{A. System Model}

In software defined SIoV, the cyber-physical world of connected vehicles is divided into three layered hierarchical network architecture including SVNs, designated control roadside units as traffic data pre-processor, and fog enabled control server (CS) as network controller (see Fig. 1). It shows the interaction between vehicular cyber and physical world with the different clusters containing different social groups of homogeneous vehicles for the data dissemination in order to meet the requirement of the SIoV. Various Road Side Units (RSUs) supporting V2I connection and V2V communication are also shown in the given scenario. Control RSUs receive the data from CS (traffic cloud) which is disseminated to the respective vehicles through common RSUs or vice versa. Highly computation oriented tasks and decision making are done at the CS. It is clarified that Internet availability is considered at control planes in the proposed architecture for software defined SIoV. The data planes consist of the local area network including vehicular resources and internet as well. By using local area vehicular networks, RSUs receive the data from all the SIOVs and utilizing the internet, RSUs forward the data to the control plane. The architecture of proposed SIoV in Fig. 1 encompasses with following characteristics of the fundamental vehicular networking model.

- SVN performs a set of tasks in vehicular environments $(\lambda)$ i.e., traffic sensing and communication.

- SVN can be a common vehicular nodes, a smart device attached Sensor Node (SN) in traffic environments or Control Node (CN) as designated RSUs.

- SIoV supports multipurpose sensing identifiable with unique identifier (ID) in the hierarchical networking architecture.

- CS can be deployed as a Cloud/Fog server which can communicate to all the CNs of the SIoV environments.

- SVN are dedicated to sense and forward heterogeneous traffic data via CNs and CS. 
TABLE I: NOMENCLATURE

\begin{tabular}{|l|l|}
\hline Symbols & Description \\
\hline$t$ & Number of control nodes (Control RSUs) \\
$r$ & Number of common nodes \\
$S N$ & Set of Control Nodes \\
$n$ & Set of common nodes \\
$x_{i}, y_{i}$ & Total destination nodes \\
$d$ & co-ordinates of the $i^{t h}$ node in space \\
$d-t h$ & Distance \\
$E_{e l e c}$ & distance threshold for communication \\
$\sigma_{f s}$ & Dissipated energy per bit \\
$\sigma_{m p}$ & Free space model coefficient \\
$E_{T X-S N}^{i j}$ & Multi-path fading coefficient \\
& Energy consumption for transmitting packet from $i^{t h}$ sensor \\
$E_{T X-C N}^{i j}$ & nodes to $j^{t h}$ node \\
$E_{R X}^{i j}$ & Transmission energy of CN to transmit $k$ bit data \\
$E_{D A}$ & Packet Reception energy \\
$E_{M T}$ & Energy consumption for compression and aggregation \\
$E_{D A}$ & Energy consumption of multi-cast tree \\
$D e l_{(i, i+1)}$ & Energy consumption of path $i$ \\
$V_{n}$ & Delay for packet communication from $i^{t h}$ node to $i+1_{t h}$ \\
$X_{n}$ & Velocity of $n^{t h}$ particle \\
$i t e r$ & Position of $n^{t h}$ particle \\
$X_{g-b e s t}$ & Current iteration number \\
$w$ & Position of best particle in the population \\
\hline
\end{tabular}

- Each SVN is capable to act either in sensing mode or communication mode.

- Any two vehicular nodes are connected with the symmetric communication links. A threshold value based better signal strength is considered for distance estimation between vehicles.

The proposed architecture of the Software Defined Wireless Sensor Network (SD-SIoV) comprises one Control Server (CS) and $r+t$ SDSN nodes out of which; $t$ is the number of Control Nodes (Control RSUs) and $r$ are common software defined Sensor Nodes (SNs) at the road or transport infrastructure (corresponding to OBUs and RSUs) which are able to connect with installed connecting devices in vehicles. CS is distant from the CNs and SNs. SD-SIoV can be logically represented as a connected graph $G=(C S, C N, S N)$ where $C S$ is the control server, $C N$ is the set of CNs (where $C N_{1}, C N_{2}$, $C N_{3}, \ldots, C N_{t} \in C N$ ) and $\mathrm{SN}$ is the set of SNs (where $S N_{1}, S N_{2}, S N_{3}, \ldots, S N_{r} \in S N$ ). Here, the problem is to construct an energy efficient (green) multi-cast tree to transfer the recurrent data from the CS to a set of destination nodes i.e., $\mathrm{D}=\left\{d_{1}, d_{2}, d_{3}, \cdots, d_{n}\right\}$. Each $d_{i}$ belongs to $\mathrm{SNs} / \mathrm{CNs}$ with subject to dynamically configuration of the SDSNs and re-fabrication of the communication links in a SD-SIoV of SIoVs such that the lifetime of the SD-SIoV is maximized.

\section{B. Green Computing in SIoV-The Optimization Problem}

This work focuses on energy minimization in data communication of SIoVs network. Thus, an energy efficient and architectural compatible energy estimation model is required. This work utilizes a widely adopted mathematical model of energy consumption for data communication which is based on path loss concept [6]. This model is comprised of multipath fading ( $d^{4}$ power loss) and free space $\left(E_{f s}\right)\left(d^{2}\right.$ power loss) channel models [6]. These models are based on distance $(d)$ between the transmitter and receiver entities. Let, $\left(x_{i}, y_{i}\right)$ and $\left(x_{j}, y_{j}\right)$ denote the coordinates of the transmitter $i$ and receiver $j$ respectively. Next, the Euclidean distance $(d)$ (between $i^{t h}$ transmitter and $j^{\text {th }}$ receiver i.e., $d_{i j}$ ) can be calculated as $\sqrt{\left(x_{j}-x_{i}\right)^{2}-\left(y_{j}-y_{i}\right)^{2}}$. Further, the power control mechanism is used to compensate this path loss. Usage of the model is defined as if distance is less than a threshold value $\left(d_{t h}\right)$, free space energy model is applied; otherwise, multipath energy model is used. To calculate the energy consumption of the network, energy model used in [21] is utilized. If a sensor node $i$ transmits $\mathrm{k}$-bit long packet to node $j$ then energy consumption will be,

$E_{T X-S N}^{i j}\left(k, d_{i j}\right)=\left\{\begin{array}{l}E_{\text {elec }} \times k+\sigma_{f s} \times k \times d_{i j}^{2}, i f d_{i j} \leq d_{t h} \\ E_{\text {elec }} \times k+\sigma_{m p} \times k \times d_{i j}^{4}, i f d_{i j}>d_{t h}\end{array}\right.$

where, $E_{\text {elec }}$ is the dissipated energy per bit to run the receiver or transmitter circuit which is dependent on several factors such as digital coding, filtering, modulation and signal spreading. $\sigma_{f s}$ and $\sigma_{m p}$ represent the coefficients of free-space and multipath fading models respectively.

Transmission energy of the control node $(C N)$ to transmit k-bit message can be calculated using Eq. 2,

$$
=\left\{\begin{array}{c}
E_{T X-C N}^{i j}\left(k, d_{i j}\right) \\
\left(E_{\text {elec }}+E_{D A}\right) \times k+\sigma_{f s} \times k \times d_{i j}^{2}, i f d_{i j} \leq d_{t h} \\
\left(E_{\text {elec }}+E_{D A}\right) \times k+\sigma_{m p} \times k \times d_{i j}^{4}, i f d_{i j}>d_{t h}
\end{array}\right.
$$

where, $E_{D A}$ represents the energy consumed by the control node which is consumed in data aggregation. To receive a packet of size $k$ bits, consumed energy at node $j\left(E_{R X}\right)$ will be,

$$
E_{R X}^{i j}(k)=\left(E_{\text {elec }}+E_{D A}\right) \times k
$$

where, $E_{D A}$ denotes the energy for compression and aggregation. Total consumed energy of a node will be,

$$
E_{i}=E_{T X}^{i}+E_{R X}^{i}
$$

As control server communicates with control nodes which later communicates with sensor nodes, therefore, energy consumption of CS includes all.

$$
E_{C S}=\Sigma_{j=1}^{|C N|}\left(E_{R X}^{j-C S} k \Sigma_{i=1}^{|S N|} E_{R X}^{i-j} k\right)
$$

where, $E_{R X}^{j-C S}$ denotes the energy consumed for communicating with member CNs and $E_{R X}^{i-j}$ denotes the consumed energy for the communication between $j^{\text {th }} \mathrm{CN}$ and $i^{\text {th }} \mathrm{SN}$.

Thus, total energy of a single cluster $j$ is

$$
E_{\text {cluster }, j}=\Sigma_{i=1}^{|S N|} E_{S N_{i}}+E_{C N_{j}}
$$

The average residual energy of SNs can be calculated using Eq. 7

$$
E_{\text {avg-re-SN }}=\frac{\sum_{i=1}^{n} E_{r e S N}}{t}
$$

Where, $E_{r e S N}$ is the residual energy of SNs installed in vehicles. Similarly, ratio of the average distance between the SNs and control RSUs, and between control RSUs and CS can be calculated as:

$d_{S N-C N}=\frac{\sum_{j=i}^{n C N} \sum_{i=1}^{n S N} \sqrt{\left(x_{C N j}-x_{S N i}\right)^{2}+\left(y_{C N j}-y_{S N_{i}}\right)^{2}}}{n S N}$ 


$$
d_{C N-C S}=\frac{\sum_{i=1}^{n C N} \sqrt{\left(x_{C N i}-x_{C S}\right)^{2}+\left(y_{C N i}-y_{C S}\right)^{2}}}{n C N}
$$

where, $x_{N O D E}$ and $y_{N O D E}$ represent the $\mathrm{X}$ and $\mathrm{Y}$ coordinates of the respective node "NODE", and $n T Y P E$ demonstrates the number of nodes of node type "TYPE". Further, the energy consumed in data transmission between the SNs and control RSUs and between control RSUs and CS can be calculated by Eqs. 10-11.

$$
\begin{gathered}
E_{S N-C N}=\frac{\sum_{j=1}^{n C N} \sum_{i=1}^{n S N j} E_{T X-S N}\left(k, d_{i, j}\right)}{n S N} \\
E_{C N-C S}=\frac{\sum_{i=1}^{n C N} E_{T X-C N}\left(k, d_{i, C S}\right)}{n C N}
\end{gathered}
$$

This energy model is compatible to the topology of the proposed architecture as shown in Fig. 1 and the energy model is used to calculate the energy consumption of any candidate solution. Further, the multicasting of traffic data from CS to respective vehicles which follow the path $(\mathrm{CS} \rightarrow \mathrm{CNs} \rightarrow \mathrm{SNs})$ or vice-versa. Total energy consumption for sending the data packets to all destination nodes $\left(D=\left\{d_{1}, d_{2}, \ldots d_{n}\right\}\right)$ include all paths from CS to the respective $\mathrm{SNs}$ which cover all destination nodes and form a Multicast Tree (MT). If $\mathrm{P}$ is the set of all paths in the selected MT then $P=\left\{P_{1}, P_{2}, \ldots P_{n P}\right\}$ where $n P$ is number of paths in multicast tree, and $P_{i}$ represents $i^{t h}$ path where $1 \leq i \leq n P$. As mentioned in system model, each path can be formed with the combination of SNs and CNs, and it may be designated to the CS and vice versa. Following this notion, the problem is to minimize the total energy consumption of the multicast tree which is formulated as follows:

To minimize,

$$
E_{M T}=\sum_{i=1}^{|n P|} E_{P_{i}}
$$

where,

$E_{P_{i}}=\left\{\begin{array}{l}E_{C S-C N_{i}}+E_{S N-C N_{i}}, \quad \text { if } C N=\text { neighbor }\left(S N_{i}\right) \\ E_{C S-C N_{i}}+\sum_{j \in P_{i}, j \neq C S}\left(E_{j} Z+E_{S N-C N_{j}} Z^{\prime}\right), o / w\end{array}\right.$

Subject to,

$$
\begin{gathered}
D=\left\{d_{1}, d_{2}, \ldots d_{n}\right\} \subseteq\left(P_{1} \cup P_{2} \cup \ldots P_{n P}\right) \\
E_{r e S N} \geq E_{t h_{S N}} \\
E_{r e C N} \geq E_{t h_{C N}} \\
\mid \text { cluster } \mid \leq t \\
r+t \geq(|S N|+|R S U| \subseteq P) \\
n e i g h b o r(C S) \subseteq C N \\
\operatorname{Del}_{M T} \geq \text { Del }_{t h}
\end{gathered}
$$

where,

$$
\operatorname{Del}_{M T}=\max _{1 \leq j \leq D} \sum_{i=1}^{v\left(P_{j}\right)} \operatorname{Del}(i, i+1)
$$

In Eq. 13, $E_{P_{i}}$ is the energy consumption of path i during the data communication. $Z$ and $Z$ ' represent flags, value of $\mathrm{Z}$ is 1 when two intermediate nodes of path are part of the cluster (either SN or normal RSU) and value of Z' is 1 when one between two intermediate nodes of the path is CN. In Eqs. $15 E_{r e S N}$ and $E_{r e C N}$ (residual energy of $\mathrm{SN}$ and $\mathrm{CN}$ ) have a lower bound threshold of battery of $E_{t h_{S N}}$ and $E_{t h_{C N}}$ respectively, which ensures that at any time if node participates in communication, it should have minimum battery to operate. In Eqs. 16 and 17, $t$ and $r$ represent the maximum number of control nodes and normal nodes of the SIOV Network respectively. In Eq. 19, $D e l_{M T}$ is the total delay for delivering the packet to all the destination of the tree, and $D e l_{t h}$ is the delay threshold and $D e l_{M T}$ is calculated using Eq. 20. $v\left(P_{j}\right)$ represents the node sequence of path for destination node $j$ from CS. Del $(i, j)$ is the delay incurred for the communication at any time between node $i$ and $j$. The constraint in Eq. 19 ensures that the delay incurred should be less than the given threshold value of delay to meet the real time application requirement.

\section{Two-way PSO-A Green Computing Solution for SIoV}

In this section, Particle Swarm Optimization [22] is enhanced by analysing the problem requirements, and utilizing the same particle as the two different solutions which is referred as Two Way Particle Swarm Optimization (TWPSO) algorithm. It employs the forward (original) particle and backward (reverse of it) particle as the two different solutions of the same particle, best of both will be accounted as Pbest solution of the particle. An effective fitness function is proposed to offer an effective multicast clustering solution in SIoV software defined architectural scenario. The convergence of the PSO is improved by proposing an adaptive inertia weight tuner. The pseudo-code for the same is given in Algorithm 1.

Explanation of Algorithm 1: The proposed algorithm begins with the predefined $N$ number of randomly generated particles; $X_{n}^{m}(t)$, and $V_{n}^{m}(t)(1 \leq n \leq N)$ are the position and velocity of $n^{t h}$ particle at $t^{t h}$ iteration in $m^{t h}$ dimension respectively. If it is not the $0^{\text {th }}$ iteration, in each iteration, the inertia weight i.e., $\omega$ is adaptively tuned by the proposed adaptive inertia weight tuner as defined in Eq. 26. With $\omega$, $V_{n}^{m}(t), X_{n}^{m}(t)$ of each particle are updated using Eqs. 23 \& 24 respectively. Further, a random number $\left(r V a r_{n}\right)$ is associated with each $n^{\text {th }}$ particle aimed to decide a pre-specified range of minimum $\left(\min _{C N}\right)$ and maximum $\left(\max _{C N}\right)$ control nodes. With this, initial $r \operatorname{Var}_{n}$ elements of each particle is accounted as number of CNs, where, number of control nodes can be changed dynamically based on traffic load. Particle's position and velocity vectors are in the form of continuous random values between two (minimum and maximum) pre-specified ranges. The Smallest Position Value (SPV) rule (Section $5.2)$ is applied to $X_{n}(t)$ vector of continuous values, and a respective discrete value vector $S_{n}(t)$ is generated. Next, $S_{n}(t)$ is evaluated using the proposed fitness function (Section 5.3) considering $r V a r_{n}$ number of CNs. As discussed, the proposed PSO algorithm acts in two ways, thus, utilizing same fitness function, each particle is evaluated in two-different 


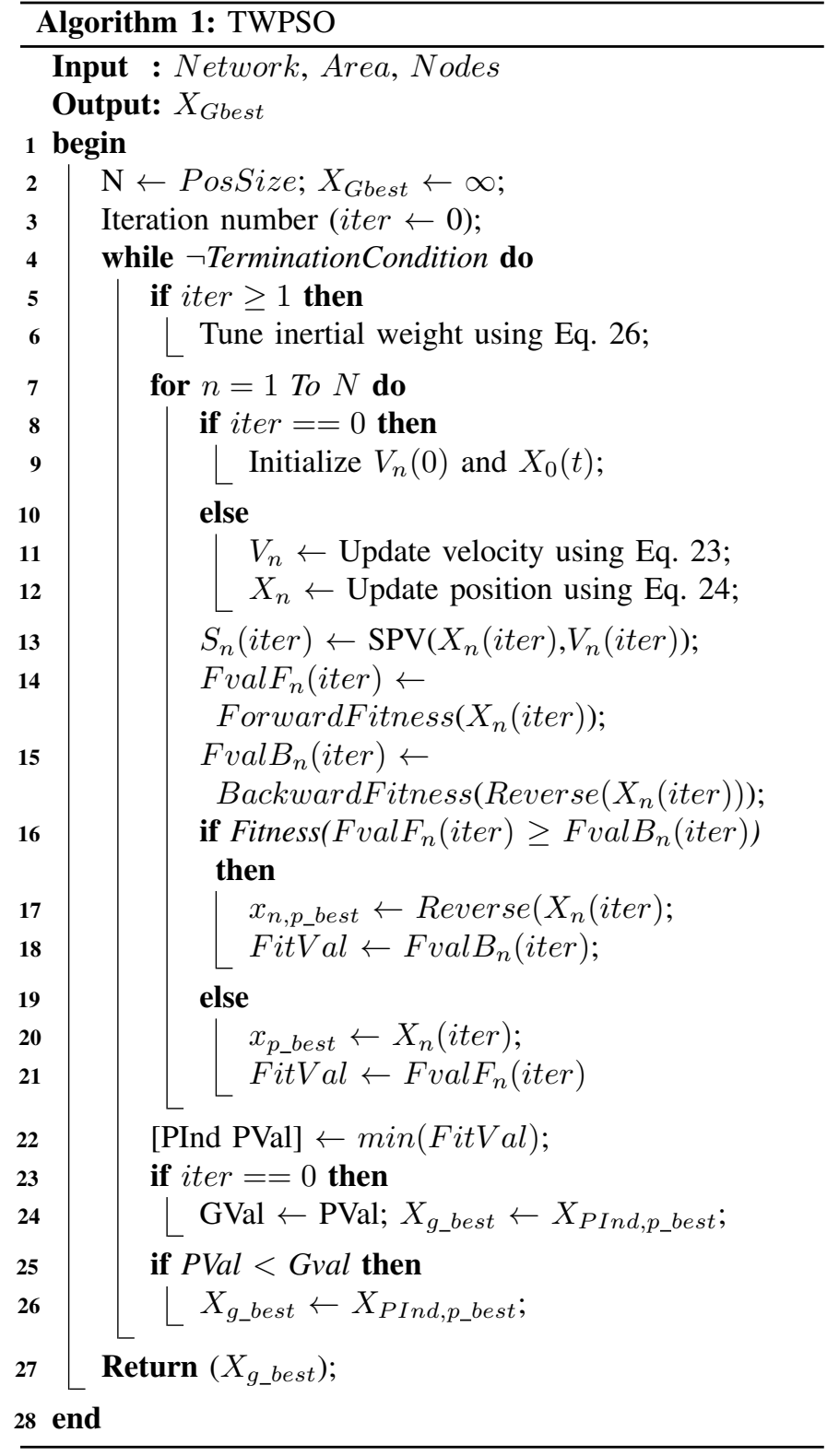

ways; one is ForwardFitness which is used to calculate the fitness value by considering initial $r V a r_{n}$ nodes as the $\mathrm{CNs}$, and another one is used to calculate the fitness value by considering reverse order $\left(r \operatorname{Var}_{n}\right)$ nodes as CNs. Based on that best of both fitness values of $n^{t h}$ particle i.e., FitVal, respective order of position vector of each particle is utilized as the new particle for the next subsequent iterative procedure. Based on the fitness value, personal best (Pbest) and Global best (Gbest) of the swarm are initialized for the case of $0^{t h}$ iteration, otherwise, Pbest and Gbest are updated as specified in algorithm 1. After each iteration, the two termination criteria of TWPSO are verified to stop procedure:i) maximum number of iterations, ii) all particles convergence to the same point. At the end, Gbest is returned as the final solution of the problem.

1) Particle Encoding and Updation: A particle consists of its respective position and velocity vector, the length of vector is equal to the number of nodes $(|S N s|+|C N s|=S D S N s)$ in the network. Let, the position and velocity of $n^{\text {th }}$ particle in $m^{\text {th }}$ dimensional search space is denoted by the position vector i.e., $X_{n}=X_{n}^{1}, X_{n}^{2}, X_{n}^{3}, \ldots . ., X_{n}^{m}$ and velocity vector i.e., $V_{n}=V_{n}^{1}, V_{n}^{2}, V_{n}^{3}, \ldots \ldots, V_{n}^{m}$. The minimum and maximum ranges of position vector $\left[X_{\min }, X_{\max }\right]$ and velocity vector $\left[V_{\min }, V_{\max }\right]$ for each particle are defined using the periphery of search space. These ranges in the planned model are anticipated to be $[0.0,4.0]$ and $[-4.0,4.0]$ for the position vector and velocity vector respectively. At $0^{t h}$ iteration, position vector corresponding to the $m^{\text {th }}$ dimension is generated by Eq. 21 .

$$
X_{n}^{m}(0)=X_{\min }+\left(X_{\max }-X_{\min }\right) * r
$$

where, $r$ variable signifies uniform random distribution between 0 and 1 . Likewise, initial velocity vector is randomly generated using the following Eq. 22.

$$
V_{n}^{m}(0)=V_{\text {min }}+\left(V_{\text {max }}-V_{\text {min }}\right) * r
$$

Further, in $0^{\text {th }}$ successive iterations of the PSO, the velocity and position vectors of the particles are updated [23] using Eq. 23 and Eq. 24 respectively.

$$
\begin{gathered}
V_{n}^{m}(\text { iter })=\omega \times V_{i}(\text { iter }-1)+c_{1} \times r_{1}(\text { Pbest } i(\text { iter }-1) \\
\left.-X_{i}(\text { iter }-1)\right)+c_{2} \times r_{2}\left(\text { Gbest }-X_{i}(\text { iter }-1)\right) \\
X_{n}^{m}(\text { iter })=X_{i}(\text { iter }-1)+V_{i}(\text { iter })
\end{gathered}
$$

where iter represents the current iteration, Pbest and Gbest represent local and global best particles respectively. $r_{1}$ and $r_{2}$ are the random variables and $c_{1}$ and $c_{2}$ are the constants used to maintain the tradeoff between exploration and exploitation. To understand the encoding in a better way, consider the example of a scenario of SIoV (as shown in Fig.1). That is consisting of scattered road lane infrastructure, upon which multi-functional sensor nodes are laid with heavy traffic load i.e., 24 vehicles which are communicating with 15 software defined RSUs sensing units out of which 5 control RSUs formed the clusters. The encoding for the same is shown in Table 1. The first/last (as two way approach) 5 nodes of the particle represent the control RSUs and the rest of the nodes are considered as common nodes which are connected to anyone of the 5 control RSUs. The nodes in a particular cluster are subjected to change after certain transmissions depending upon their residual energy content. Thus, the number of control RSUs and SNs have dynamic behaviour during the data transmission. Therefore, the problem is to form optimal clusters for the data routing considering dynamism with the objective of minimized energy consumption and delay in data communication.

2) Smallest Position Value: As discussed, initial encoding of the particles is in continuous values vector, however, solution is required to be in the form of discrete value vector. Therefore, there is need to convert continuous values vector into discrete values vector. To do this, Smallest Position Value (SPV) operator is employed. SPV [24] is a heuristic rule which is used to convert the continuous value vector into discrete value vector for all class of sequencing problem. Same is utilized with the PSO which enables the conversion of continuous position vector $X$ of the particle 
TABLE II: Initial encoding and velocity of a particle

\begin{tabular}{llllllllllllllll}
\hline $\mathrm{K}$ & 1 & 2 & 3 & 4 & 5 & 6 & 7 & 8 & 9 & 10 & 11 & 12 & 13 & 14 & 15 \\
\hline $\mathrm{X}$ & 1.83 & 2.1 & 4.7 & 1 & 0.79 & 2 & 1.56 & 4 & 0.51 & 4.1 & 1.61 & 3.62 & 2.85 & 3.02 & 3.99 \\
$\mathrm{~V}$ & -1.02 & 4 & -0.2 & 3 & -1 & -0.28 & 3.69 & -1.9 & 4.99 & -3.8 & -0.35 & 1.72 & -2.9 & -3.9 & 1 \\
\hline
\end{tabular}

into the discrete valued position vector $S$. It uses a $\operatorname{sort}()$ function which arranges the particle's dimension indexes in an increasing order w.r.t. position to produce the discrete value vector corresponding to its continuous position vector.

3) Fitness Function: In order to maximize the lifetime of the network, a novel fitness function is proposed through selection of the most competent clustered tree for multicast routing in SIoV. The formation of clusters includes the selection of optimized number of control RSUs and the selection of the location of respective control RSUs in SD-SIoV. In the proposed fitness function, the inclination is towards selecting control RSUs with greater residual energy and minimum distance from the CS and destination nodes. In order to meet our requirements towards a novel fitness function, the fitness function is composed of three sub components i.e., $f_{1}, f_{2}$ and $f_{3} . f_{1}$ denotes the ratio of average residual energy of control RSUs to other SNs. Maximizing $f_{1}$ signifies the selection of higher residual energy for control RSUs. $f_{2}$ denotes the ratio of the average distance between the SNs and control RSUs, and between control RSUs and CS which can be calculated using Eq. 8 and Eq. 9 respectively. Further, $f_{3}$ component of the fitness function emphasizes towards maximizing number of destination nodes in a same cluster to minimize energy consumption in data routing. Each sub-component $f_{1}, f_{2}$ and $f_{3}$ of the fitness function is accompanied by a constant $\alpha, \beta$ and $\gamma$ respectively, where, $\alpha+\beta+\gamma=1$. In our experiments, equal weight to all components offer good results. For the selection of control RSUs with greater residual energy and balanced trade-off between distance and energy, a combined and novel fitness function is formulated in Eq. 25.

$$
\begin{array}{r}
F=\alpha f_{1}+\beta f_{2}+\gamma f_{3} \\
=\alpha \frac{E_{\text {CNTOCS }}}{E_{\text {SNTOCN }}}+\beta \frac{1}{\frac{d_{S N T O C N}+d_{C N T O C S}}{2}}+\gamma \frac{1}{\mid \text { clusters } \mid}
\end{array}
$$

where, $\alpha+\beta+\gamma=1$, the default values for the $\alpha, \beta$ and $\gamma$ are $0.5,0.25$ and 0.25 respectively. As the main objective of this work is to obtain green communication, therefore, higher weight-age is given to $\alpha$.

4) Inertia weight: Inertial weight $(\omega)$ plays a very significant role in the convergence of the PSO algorithm. During initialization of PSO, particles are randomly scattered in multidimensional search space and in the successive iterations, they re-defines their positions following the PSO rules during the run with the help of $\omega$. The balance between local search and global search is controlled by the inertia weight $\omega$ to determine optimal solution. It's increasing value contributes to global search whereas its decreasing value denotes its inclination towards local search. Following the adaptive $\omega$ tuner (Eq. 26), an effective trade-off is maintained between exploration and exploitation by accounting successive iteration number.

$$
w=w_{\max }-\frac{w_{\max }-w_{\min }}{\text { iter }_{\max }} \times i \text { iter }
$$

where $w_{\max }$ and $w_{\min }$ are the maximum and minimum limits of the inertia weight respectively. iter denotes the current iteration number and iter $_{\max }$ maximum number of iterations. This inertia function helps to control the current velocity by considering past velocity. Moreover, this function provides better performance with the proposed fitness function.

5) Cluster formation: Once, the control nodes are selected through the solution (Gbest) offered by the proposed TWPSO algorithm, each control RSU introduces itself to the network by broadcasting a minor advertisement message (ADV). It makes use of a non-persistent carrier-sense multiple access (CSMA) MAC protocol. The ADV message composed of control RSU's ID and a header that differentiates advertisement message. Each common node determines its cluster by choosing the control RSU that entails the minimum data transmission energy utilizing the strength of the ADV message. Once, each common node has decided as to which cluster it belongs, it must inform other control RSUs of its decision by transmitting a LINK-REQ message. This message is also a short message which consists of node's ID, the belonging control RSU's ID and the sender's residual energy. In this way, the clusters are formed and the responsibility of each node in the network is determined. Control RSU in a cluster acts as the control head which collects the data from other SNs and aggregate it then transfer to CS or vice-versa. This way, clusters setup phase is completed, and the data transmission phase begins with the determined topology [18].

\section{CAse Study}

\section{A. Parameter Settings}

All experiments were conducted on Intel i5 processor with 4GB RAM in Matlab tool. For the simulation, different OBUs based vehicles and RSUs were generated in the given area using various distribution methods in order to analyze the performance of TWPSO in sparse and dense vehicular networks. For the experimentation, number of destination nodes, initial energy of nodes, number of control RSUs and number of vehicles were varied. Meanwhile, for TWPSO parameters, results are collected for the varied population size and number of iterations. Experimental evaluation also includes the results for number of transferred packets to control RSUs, sum of energy and number of rounds. The range, [minimum maximum] of TWPSO parameters such as population and number of iterations are varied in range 30-80 and 5-50 respectively. Particles were randomly generated in multidimensional search space where position and velocity vectors of particles lie in interval $[0,4]$ and $[-4,4]$ respectively as in [24]. To verify the 
TABLE III: Sensitivity of PSO Parameters in TWPSO

\begin{tabular}{|cccccc|}
\hline Case & $w_{\max }$ & $w_{\min }$ & $C_{1}$ & $C_{2}$ & Fitness value \\
\hline 1 & 0.3 & 0.1 & 0.5 & 2.0 & 5.106 \\
2 & 0.5 & 0.1 & 0.5 & 2.0 & 5.606 \\
3 & 0.3 & 0.1 & 1.0 & 2.0 & 5.105 \\
4 & 0.5 & 0.2 & 1.0 & 2.0 & 4.604 \\
5 & 0.7 & 0.2 & 0.5 & 1.5 & 4.602 \\
6 & 0.7 & 0.4 & 1.0 & 1.5 & 5.106 \\
7 & 0.5 & 0.2 & 1.5 & 1.0 & 4.602 \\
8 & 0.3 & 0.2 & 2.0 & 0.5 & 5.105 \\
9 & 0.9 & 0.4 & 0.5 & 2.0 & 5.104 \\
$\mathbf{1 0}$ & $\mathbf{0 . 9}$ & $\mathbf{0 . 2}$ & $\mathbf{2 . 0}$ & $\mathbf{2 . 0}$ & $\mathbf{5 . 6 1 2}$ \\
11 & 0.9 & 0.2 & 1.5 & 2.0 & 5.103 \\
12 & 0.5 & 0.1 & 2.0 & 2.0 & 5.1 \\
\hline
\end{tabular}

effect of TWPSO parameters, different training experiments were conducted for inertia weight, cognitive parameter and social parameter. Table 2 shows different combinations of these parameters and the performance of TWPSO in terms of fitness. For each input combination, algorithm's behavior is observed, and best fitness producing combination is selected. From Table 2, it can be concluded that case $10\left(C_{1}=2.0, C_{2}\right.$ $=2.0, w_{\max }=0.9$ and $\left.w_{\min }=0.2\right)$ offers better results.

As algorithm behaves stochastically, therefore, a statistical test is performed to evaluate the significance of the obtained results. Mean and standard deviation compared the overall performance whereas statistical test considers the results of each run to prove the significance of the results. The KruskalWallis H Test (non-parametric) [25] is conducted over the obtained results of the fitness for $\alpha=0.5$. Three scenarios of the network were considered to solve the given problem including 100, 200 and 500 nodes respectively. Test metrics are given in Table 3, and obtained $H_{S T A T}$ using the test is 8.5 which is greater than 5.9 (critical value). Therefore, the three probabilities are distributed as same. Similarly, test is done over similar scenarios and initialization parameters for NWPSO. Mean Time To Failure (MTTF) of the network is one of the metrics for performance measurement.The performance of TWPSO is compared with NWPSO [18] and LEACH [26] algorithms to verify its effectiveness.

\section{B. Green Computing Efficiency}

Swarm based optimization algorithms have an influential parameter named as diversity as it quantifies the exploration and exploitation [23]. For demonstrating the correct behavior of the algorithm, diversity measures the particle's dispersion. Diversity accurately quantifies the search behaviour of the swarm in terms of exploration and exploitation with in the boundaries of the search space. It provides the results over various courses of the iterations. Consideration of each variation of the diversity measure is not possible, thus, Euclidean distance based metric is utilized for the same. Whenever normalization is needed then diameter is used. Network scenarios of the Poisson distribution are deployed for performance measurements. These metrics are defined as follows:

Swarm diameter and radius: Maximum distance between any two particles of the swarm is termed as the diameter of the swarm which is calculated as follows:

$$
|D|=\max _{(j \neq i) \in[1,|S|]}\left(\sum_{k=1}^{K} \sqrt{\left(x_{j k}-x_{i k}\right)^{2}}\right)
$$

where, $|S|$ represents total swarms and k represents dimensions of the stated problem, and $x_{j k}$ shows the position of $k^{t h}$ dimension of $j^{\text {th }}$ particle. The radius of the search space of the swarm can be calculated as the distance between the center of the swarm and farthest particle from it. Diameter is used to measure the diversity.

Average Distance around the center of Swarm: This metric helps to calculate the diversity of the swarm. Higher value indicates high dispersion of the particles around center and lower value indicates that the swarm convergence around the center.

$$
D_{c}=\frac{1}{|S|} \max _{j \in[1,|S|]}\left(\sum_{k=1}^{K} \sqrt{\left(x_{j k}-\bar{x}_{j}\right)^{2}}\right)
$$

where, $\bar{x}_{j}^{2}$ shows the mean value of $j^{\text {th }}$ particle position.

The Normalized Average Distance around the Swarm Center: It can be calculated as:

$$
D_{n}=\frac{1}{|S| \cdot K} \max _{j \in[1,|S|]}\left(\sum_{k=1}^{K} \sqrt{\left(x_{j k}-\bar{x}_{j}\right)^{2}}\right)
$$

Fig. 2 illustrates the performance of the proposed algorithm in terms of swarm diameter, average distance around the swarm, normalized average distance around the swarm and swarm fitness for 50-1000 iterations. Fig. 2a, fig. 2b and fig. 2c show the aforementioned results for 50 nodes, 100 nodes and 500 nodes plotted against the iteration numbers. It illustrates the swarm diversity which is returned by the normalized average distance present around the swarm center relative to best particle's fitness.

\section{Green Computing Benefits}

In order to make the fair comparative study, a set of experiments are conducted on the same set of parameters as mentioned in Section 6.1. To observe the behavior of TWPSO over different population size and number of iterations, a set of experiments are conducted with different combinations of these parameters which are mentioned with the respective results. From the Fig. 3, it is inferred that the TWPSO achieves optimal fitness value at 30 iterations and 50 population size. Thus, combination of 30 iterations and 50 particles is considered for rest of the experiments as fitness is maximum at this combination that is desired. Once, the population size and number of iterations are fixed, the performance of TWPSO is compared with NWPSO and LEACH algorithms to verify its effectiveness.

Fig. 4a presents the results in terms of MTTF on varied number of control RSUs which are shown on $\mathrm{X}$ axis of the plot, and rest of the parameters are set as default parameters as mentioned in Section 6.1. From the results, it is observed that the TWPSO algorithm outperforms other state of the art algorithms when number of control RSUs varies; it proves the scalability feature of the TWPSO. This is due to the 


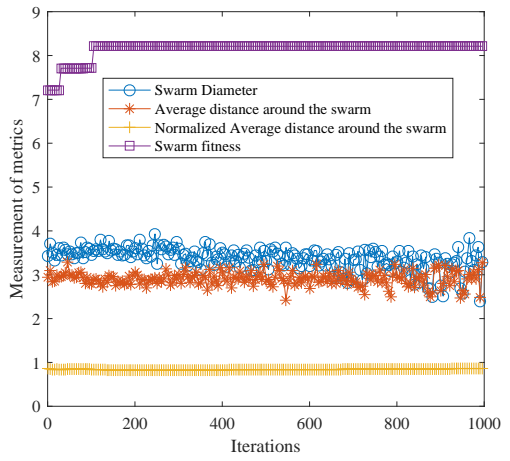

(a) 50 vehicles scenario

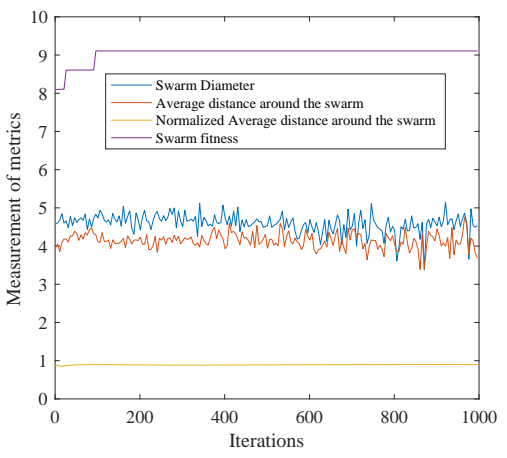

(b) 100 vehicles scenario

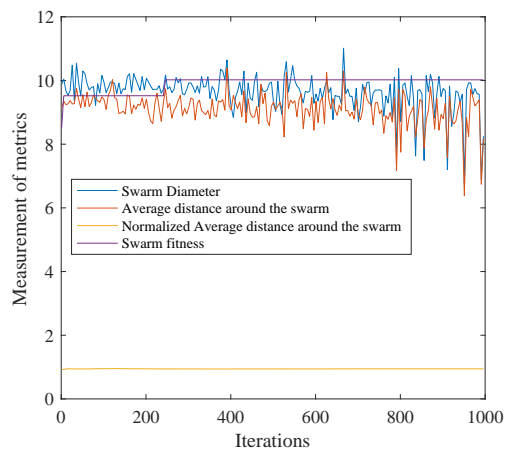

(c) 500 vehicles scenario

Fig. 2: Normalized Average Distance for different scenarios with respect to Fitness, Diameter and Average Distance

TABLE IV: Fitness Values of TWPSO on 20 runs for Kruskal-Wallis H test

\begin{tabular}{|c|c|c|c|c|c|c|c|c|c|c|c|c|c|c|c|c|c|c|c|c|}
\hline Number of runs & 1 & 2 & 3 & 4 & 5 & 6 & 7 & 8 & 9 & 10 & 11 & 12 & 13 & 14 & 15 & 16 & 17 & 18 & 19 & 20 \\
\hline N/W Scenario1 100 Nodes & 7.51 & 7.51 & 8.01 & 7.01 & 6.01 & 6.01 & 7.01 & 7.01 & 7.01 & 6.51 & 7.01 & 5.51 & 6.51 & 8.51 & 7.01 & 7.01 & 8.01 & 7.01 & 7.01 & 7.01 \\
\hline N/W Scenario2 200 Nodes & 6.49 & 4.99 & 5.49 & 4.99 & 6.5 & 5.99 & 4.99 & 6.5 & 8.5 & 7 & 5.48 & 6.49 & 6.5 & 8.49 & 6.99 & 5.5 & 5.49 & 6 & 6.5 & 8 \\
\hline N/W Scenario3 500 Nodes & 8.36 & 6.9 & 6.39 & 7.38 & 6.37 & 6.42 & 7.38 & 6.39 & 6.39 & 6.37 & 6.87 & 7.89 & 6.39 & 6.39 & 8.38 & 6.88 & 6.39 & 7.39 & 6.38 & 7.39 \\
\hline
\end{tabular}

TABLE V: Comparative results of TWPSO Fitness

\begin{tabular}{|l|l|l|l|l|l|l|}
\hline \multirow{2}{*}{ Network scenario } & \multicolumn{2}{|c|}{ TWPSO } & \multicolumn{2}{c|}{ NWPSO } & \multicolumn{2}{c|}{ LEACH } \\
\cline { 2 - 7 } & Mean & STD & Mean & STD & Mean & STD \\
\hline 50 & 8.06 & 0.17 & 6.56 & 0.16 & 6.05 & 0.10 \\
\hline 100 & 7.06 & 0.63 & 7.06 & 0.42 & 6.55 & 0.31 \\
\hline 200 & 7.56 & 0.74 & 6.74 & 0.34 & 6.03 & 0.32 \\
\hline 300 & 7.53 & 0.52 & 6.14 & 0.46 & 6.35 & 0.34 \\
\hline 400 & 8.56 & 0.76 & 8.06 & 0.45 & 6.05 & 0.42 \\
\hline 500 & 7.43 & 0.64 & 7.12 & 0.51 & 6.86 & 0.52 \\
\hline 600 & 7.82 & 0.72 & 6.41 & 0.42 & 6.14 & 0.21 \\
\hline
\end{tabular}

fact of effectiveness of the proposed fitness function and the two-way implementation approach of the PSO algorithm. Following this, TWPSO optimizes the cluster formation with minimal energy consumption in data communication which results in higher MTTF. Further, to verify the effectiveness of the algorithm on varied number of destinations (target vehicles), a set of experiments are conducted and results are presented in Fig. 4b. Fig. 4b shows the lifetime of the network for different set of destinations varying between 5 and 40 for a VANET of 100 nodes. From the results, it is analyzed that despite of increasing number of targeted vehicles (for data dissemination), MTTF of the network remains balanced even when load on control RSUs increases. Reason for the same is that TWPSO focuses on clustering of control RSUs among the destinations such that more number of destinations becomes member of the same cluster. This helps to decrease the number of control packet exchanges, and thus contributes toward increasing network lifetime. This is due to the fitness function which is technically designed for the same objective. It evidences the significance of the proposed fitness function.

As OBUs/RSUs communication devices are battery operated and battery life of these devices affected with the size of vehicular networks. Thus, there is need to analyze the behavior of the algorithm for different number of vehicular networks. Thus, to evaluate the performance of the algorithm on different size of vehicular networks, a set of experiments are conducted by varying the number of vehicles between 300 and 600. In order to conduct this set of experiments, except

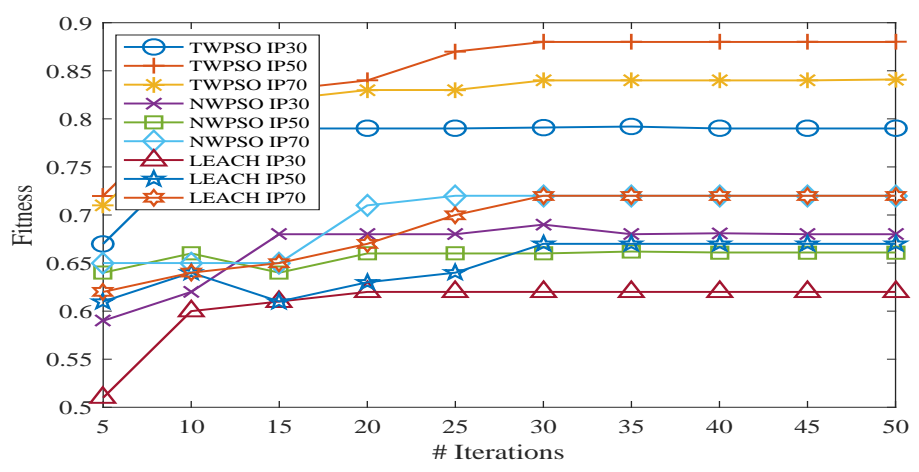

Fig. 3: Influence of the PSO Parameters on TWPSO

the number of vehicular nodes, all the testing parameters are taken as the default. Fig. $4 \mathrm{c}$ demonstrate the pronged lifetime of the network on different network scenarios as the algorithm minimizes the number of message exchange between control RSUs and control server by preventing irrelevant RSUs to communicate with the server. As a result, the energy is conserved which can be seen in Fig. 4c.

In SDN enabled environment, few RSUs are selected as the control nodes which communicate with their member RSUs and vehicles. As all the data packets of the respective cluster are forwarded through the respective control RSU only, this way, a huge amount of energy is consumed in data communication. With the advent of technology, it is allowed that communication can take place even if few RSU units have less energy by skipping high distant transmission through them. Thus, with time, control RSUs will also be re-elected, but it will result in several packet exchanges, therefore, there is need to select the optimal control RSUs. In order to simulate this, we conducted a set of experiments in which nodes are equipped with initial energy between $0.5 \mathrm{~J}$ to $2.0 \mathrm{~J}$ as shown in Fig. 4d. From the results (Fig. 4d), it can be observed that MTTF for TWPSO is better than NWPSO at high initial energy as well as at low initial energy availability. Further, it can be observed that with the increased battery power, lifetime 


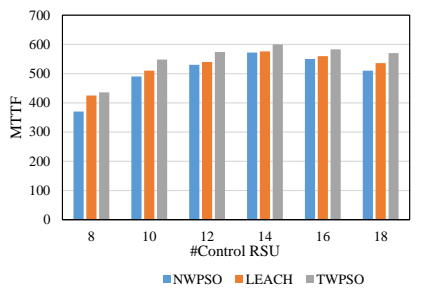

(a) Different clusters of SVN

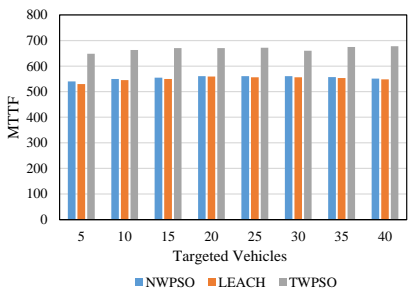

(b) Different receiving SVN

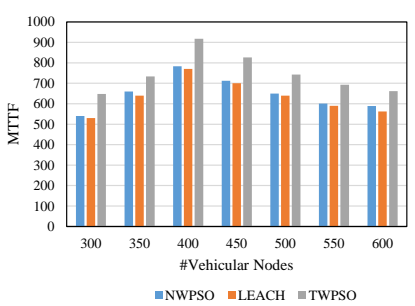

(c) Different vehicular network size

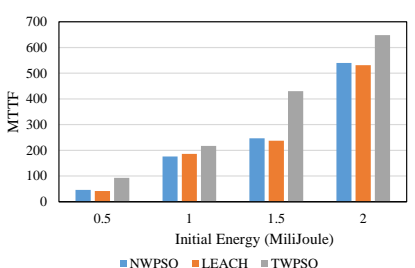

(d) Different initial energy of SVN

Fig. 4: Comparative performance analysis of TWPSO with the state-of-the-art models

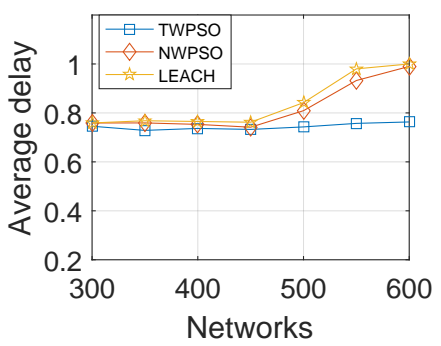

(a) Average Delay

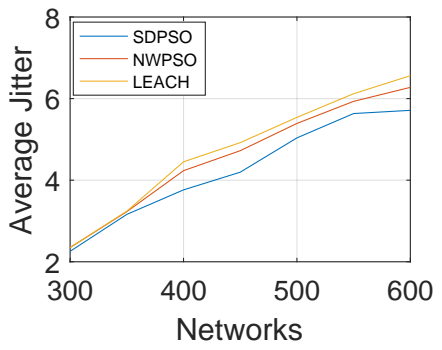

(b) Average Delay Jitter
Fig. 5: Comparative performance considering delays with vehicular network size

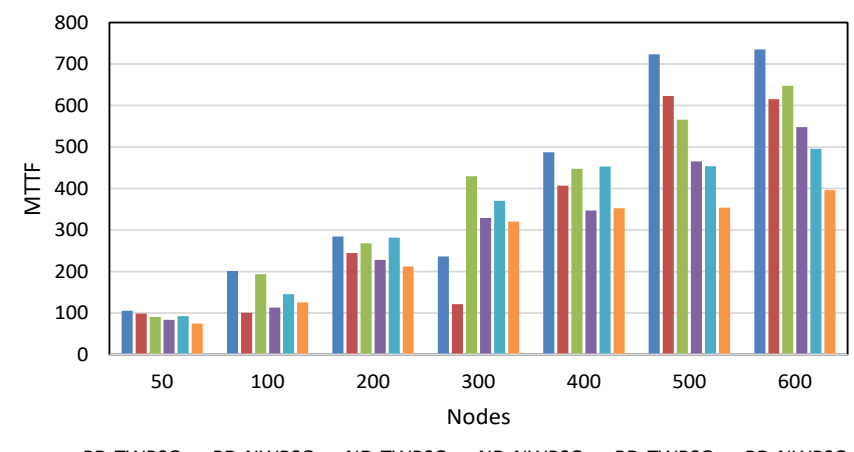

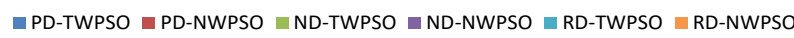

Fig. 6: MTTF comparison for different distributions

of nodes increases which helps the network to work for longer period without any failure. Thus, nodes the with high battery power are selected for multicasting as the control nodes.

In order to test the stability in the performance, the proposed algorithm is compared with NWPSO and LEACH on 100 runs for each test instance. The results are shown in Table 4 on different network scenarios with mean and standard deviation (STD). From the Table 4, it can be observed that the mean of fitness is better for TWPSO than other two comparative algorithms. The reason for the same is that the proposed algorithm searches for the solution in both directions with the balanced trade-off between exploration and exploitation. Further, to analyze the network performance for delay and delay jitter metrics, results have been compared with NWPSO for various network size. Fig. 5a shows that delay in communication for TWPSO is lesser than the delay for other algorithms. Formation of clusters helps to reduce the load on network as control RSUs take care of message dissemination among vehicles, which helps to reduce the delay. Further, TWPSO handles the issue of loop formation with less computation, and it selects lesser control RSUs in the route formation. Thus, irrelevant nodes for cluster formation are ignored which help in reducing delay for the data transmission to the targeted destination vehicles. Average jitter throughout the transmission is proportional to delay; therefore, delay jitter for small delay is also less which can be observed in Fig. 5 b.

In vehicular networks, a set of experiments are conducted on three types of node distributions in vehicular network: Random Distribution (RD), Poisson Distribution (PD) and Normal Distribution (ND), and results are compared with its counterpart algorithm (NWPSO). A set of vehicular networks are randomly generated on various network scenarios by varying the number of nodes between 50 and 600. As node distributions strategy affects the performance of the algorithm, thus, to test the distribution stability of the algorithm, this set of experiment is conducted. Fig. 6 shows that the MTTF of the network following these distributions is compared, and results indicate that MTTF of TWPSO is higher for all the scenarios in comparison to NWPSO for the given node densities.

\section{CONCLUSION}

This work explored software defined architecture for SIoV data communication with the objective of life-time maximization of Software Defined-Social Internet of Vehicle (SD-SIoV). In order to offer dynamic multi-cast routing utilizing SDSIoV architecture, a Two-Way PSO (TWPSO) meta-heuristic algorithm is presented. TWPSO is an enhanced form of PSO. With TWPSO, CNs are selected to handle dynamic tasks which are controlled through CS. As an experimental evidence, TWPSO outperforms state-of-the-art techniques and significantly maximizes the life-time of the network with minimum delay for data transmission. This protocol can be a good candidate for the future SIoV's green data communication. In future, the proposed SD-SIoV architecture will be explored to enabled smart traffic services on-road environments.

\section{REFERENCES}

[1] N. Kumar, S. S. Rahman, and N. Dhakad, "Fuzzy inference enabled deep reinforcement learning-based traffic light control for intelligent transportation system," IEEE Transactions on Intelligent Transportation Systems, 2020.

[2] T. A. Butt, R. Iqbal, S. C. Shah, and T. Umar, "Social internet of vehicles: Architecture and enabling technologies," Computers \& Electrical Engineering, vol. 69, pp. 68-84, 2018.

[3] R. Kasana, S. Kumar, O. Kaiwartya, R. Kharel, J. Lloret, N. Aslam, and T. Wang, "Fuzzy-based channel selection for location oriented services in multichannel vcps environments," IEEE Internet of Things Journal, vol. 5, no. 6, pp. 4642-4651, 2018. 
[4] A. J. Kadhim and S. A. H. Seno, "Energy-efficient multicast routing protocol based on sdn and fog computing for vehicular networks," Ad Hoc Networks, vol. 84, pp. 68-81, 2019.

[5] Y. Cao, H. Song, O. Kaiwartya, B. Zhou, Y. Zhuang, Y. Cao, and $\mathrm{X}$. Zhang, "Mobile edge computing for big-data-enabled electric vehicle charging," IEEE Communications Magazine, vol. 56, no. 3, pp. 150-156, 2018.

[6] S. Rho and Y. Chen, "Social internet of things: Applications, architectures and protocols," 2019.

[7] S. F. Hasan, X. Ding, N. H. Siddique, and S. Chakraborty, "Measuring disruption in vehicular communications," IEEE Transactions on Vehicular Technology, vol. 60, no. 1, pp. 148-159, 2010.

[8] J. Guerrero-Ibáñez, S. Zeadally, and J. Contreras-Castillo, "Sensor technologies for intelligent transportation systems," Sensors, vol. 18, no. 4, p. 1212, 2018.

[9] N. Kumar and D. P. Vidyarthi, "A green routing algorithm for iot-enabled software defined wireless sensor network," IEEE Sensors Journal, vol. 18 , no. 22 , pp. 9449-9460, 2018.

[10] A. Aliyu, A. H. Abdullah, O. Kaiwartya, Y. Cao, M. J. Usman, S. Kumar, D. Lobiyal, and R. S. Raw, "Cloud computing in vanets: architecture, taxonomy, and challenges," IETE Technical Review, vol. 35, no. 5, pp. 523-547, 2018.

[11] D. Zhang, F. R. Yu, and R. Yang, "Blockchain-based distributed software-defined vehicular networks: A dueling deep q-learning approach," IEEE Transactions on Cognitive Communications and Networking, vol. 5, no. 4, pp. 1086-1100, 2019.

[12] A. Zekri and W. Jia, "Heterogeneous vehicular communications: A comprehensive study," Ad Hoc Networks, vol. 75, pp. 52-79, 2018.

[13] O. Kaiwartya, A. H. Abdullah, Y. Cao, A. Altameem, M. Prasad, C.-T. Lin, and X. Liu, "Internet of vehicles: Motivation, layered architecture, network model, challenges, and future aspects," IEEE Access, vol. 4, pp. 5356-5373, 2016.

[14] L. Zhai, X. Ma, J. Gao, and Y. Liu, "Psmr: publish/subscribe multi-cast routing for wireless ad hoc networks," in 2007 International Conference on Computational Intelligence and Security Workshops (CISW 2007). IEEE, 2007, pp. 554-557.

[15] M. Zhu, J. Cao, D. Pang, Z. He, and M. Xu, "Sdn-based routing for efficient message propagation in vanet," in International Conference on Wireless Algorithms, Systems, and Applications. Springer, 2015, pp. 788-797.

[16] O. Sadio, I. Ngom, and C. Lishou, "Design and prototyping of a software defined vehicular networking," IEEE Transactions on Vehicular Technology, 2019.

[17] J. Bhatia, Y. Modi, S. Tanwar, and M. Bhavsar, "Software defined vehicular networks: A comprehensive review," International Journal of Communication Systems, vol. 32, no. 12, p. e4005, 2019.

[18] W. Xiang, N. Wang, and Y. Zhou, "An energy-efficient routing algorithm for software-defined wireless sensor networks," IEEE Sensors Journal, vol. 16, no. 20, pp. 7393-7400, 2016.

[19] P. Dai, K. Liu, X. Wu, Z. Yu, H. Xing, and V. C. S. Lee, "Cooperative temporal data dissemination in sdn-based heterogeneous vehicular networks," IEEE Internet of Things Journal, vol. 6, no. 1, pp. 72-83, 2018.

[20] M. Elhoseny, "Intelligent firefly-based algorithm with levy distribution (ff-1) for multicast routing in vehicular communications," Expert Systems with Applications, vol. 140, p. 112889, 2020.

[21] L. Farhan, R. Kharel, O. Kaiwartya, M. Hammoudeh, and B. Adebisi, "Towards green computing for internet of things: Energy oriented path and message scheduling approach," Sustainable Cities and Society, vol. 38, pp. 195-204, 2018

[22] O. Kaiwartya, S. Kumar, D. Lobiyal, P. K. Tiwari, A. H. Abdullah, and A. N. Hassan, "Multiobjective dynamic vehicle routing problem and time seed based solution using particle swarm optimization," Journal of Sensors, vol. 2015, 2015.

[23] D. Bratton and J. Kennedy, "Defining a standard for particle swarm optimization," in 2007 IEEE swarm intelligence symposium. IEEE, 2007, pp. 120-127.

[24] R. Chaudhry, S. Tapaswi, and N. Kumar, "Forwarding zone enabled pso routing with network lifetime maximization in manet," Applied Intelligence, vol. 48, no. 9, pp. 3053-3080, 2018.

[25] Y. Lou, S. Y. Yuen, and G. Chen, "Evolving benchmark functions using kruskal-wallis test," in Proceedings of the Genetic and Evolutionary Computation Conference Companion. ACM, 2018, pp. 1337-1341.

[26] G. K. Nigam and C. Dabas, "Eso-leach: Pso based energy efficient clustering in leach," Journal of King Saud University-Computer and Information Sciences, 2018.

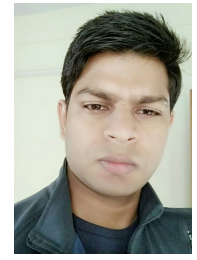

N. Kumar received his M.tech and Ph.D. degrees from Jawaharlal Nehru University, New Delhi India in 2012, 2016 respectively. He is currently working as Assistant Professor in IIT Roorkee India. He has published several research publications in worlds top tier publishers, such as IEEE journals and transactions, Elsevier, and springer journals. His research interests include Vehicular Networking, Algorithm design, and High Performance Computing.

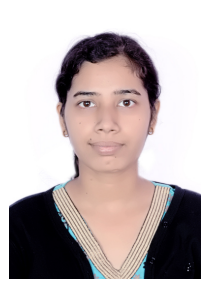

R. Chaudhry received his M.E. degree in CSE from the Indian School of Mines, Dhanbad, India, in 2014 and Ph.D. degree from the Atal Bihari Vajpayee Indian Institute of Information Technology and Management, Gwalior, India. SHe is currently working as Assistant Professor in IIIT Naya Raipur India. Her research interests include wireless sensor networks, IoT, and vehicular adhoc networks.

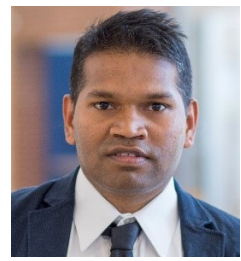

O. Kaiwartya [M 14, SM 19] is currently working as a Senior Lecturer at the Nottingham Trent University, UK. Previously, He was a Research Associate at the Northumbria University, Newcastle, UK, in 2017 and a Postdoctoral Research Fellow at the University of Technology Malaysia (UTM) in 2016. He received his Ph.D. degree in Computer Science from Jawaharlal Nehru University, New Delhi, India, in 2015. He is the Fellow in Higher Education Academy (FHEA), UK. His research interest focuses on Drone Enabled Networking, E-Mobility Centric Electric Vehicles, IoT Enabled Smart Services, Connected Vehicles, and Next Generation Wireless Systems.

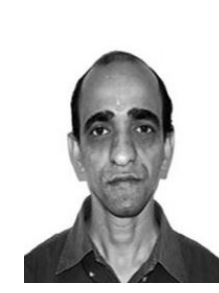

N. Kumar [M 16] received the Ph.D. degree in computer science and engineering from Shri Mata Vaishno Devi University, Katra, India in 2009. He was a Post-Doctoral Fellow from the Coventry University, Coventry, U.K. He is working as an Professor with the Department of Computer Science and Engineering, Thapar University, Patiala, India. He has authored more than 100 scholarly research publications in top journals and conferences. His research interests include vehicular cyber-physical systems, mobile cloud computing, smart grid, IoT, service oriented computing, security issues in wired/wireless networks.

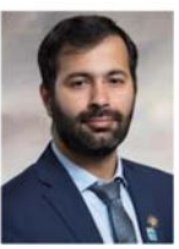

SH. Ahmed [SM 18] is currently an independent researcher. Previously, he was an Assistant Professor in the Department of Computer Science at Georgia Southern University, USA. He was a Post-Doctoral Fellow in the University of Central Florida, Orlando, USA. Before moving to the United States, he completed his Master combined Ph.D. Degree from Kyungpook National University, South Korea. He has authored over 200 international publications including Journal articles, Conference Proceedings, and 03 books. In 2016, his work on robust content retrieval in future vehicular networks lead him to win the Qualcomm Innovation Award in Korea. Hassan's research interests include Sensor and Ad hoc Networks, Cyber-Physical Systems, Vehicular Communications, and Future Internet. 